\title{
Analysis of WiMAX Performance Improvement Using Serial and Parallel Concatenated Convolutional Codes
}

\author{
P. Rastegari, M. Majidi, and M. Khalilian
}

\begin{abstract}
This paper is proposed for analysis of WiMAX performance improvement by coding and iterative decoding algorithms. Forward error correction (FEC) is one of the most important sections in physical layer of WiMAX system. IEEE802.16 standard has offered several types of FEC for WiMAX e.g. Reed Solomon and Convolutional Code (RS-CC). This paper offers using of two kinds of Turbo codes, Serial and parallel concatenated convolutional codes (SCCCs and PCCCs) with suitable parameters as FEC for WiMAX. Simulations are carried out for RS-CC, SCCC and PCCC. BPSK and 64QAM are selected as modulation schemes in simulations. Simulation results show that the use of SCCC and PCCC has better performance than RS-CC because of their superior coding and iterative decoding algorithm in this work.
\end{abstract}

Index Terms-WiMAX, SCCC, PCCC, iterative decoding algorithm.

\section{INTRODUCTION}

Worldwide Interoperability for Microwave Access (WiMAX) based on IEEE802.16 standard is proposed for broadband wireless communications over long distance [1]. Some equipment are placed in in transmitter and receiver for more reliable data transmission. For instance, coding and decoding blocks for error correction. Standard of WiMAX has offered using Reed Solomon and convolution codes (RS-CC) for coding in transmitter, and Viterbi algorithm (VA) for decoding in receiver [2].

Convolutional coding is applied for digital communication systems because it has real-time decoding with high performance. In order to increase performance of coding, a large constraint length is required. Consequently complexity of decoding increases and it is not desirable.

Code concatenation is a multilevel coding which was offered by Elias in 1950s [3], then Forney continued work on concatenated codes and proposed a way to improve error correction without increasing the decoding complexity [4]. In 1993, turbo codes were presented for remarkable performance of coding, acceptable decoding complexity, and having error correction abilities near to Shannon's limit [5]. They are classified into two groups, serial and parallel

Manuscript received September 12, 2012; revised November 27, 2012.

P. Rastegari was with the Electrical Engineering Department, Islamic Azad University, Najafabad Branch, Iran (e-mail: parisa_rastegari @ sel.iaun.ac.ir).

M. Majidi is with the Electrical Engineering, Amirkabir University of Technology, Tehran, Iran (e-mail: mmajidi@aut.ac.ir).

M. Khalilian is with the Electrical Engineering Department, Islamic Azad University, Najafabad Branch, Iran (e-mail: m.khalilian@iaun.ac.ir). concatenated convolutional codes (SCCCs and PCCCs). In addition, they are called serial and parallel turbo codes.

The organization of the paper is as follows: Section II explains about encoder structure of SCCCs and PCCCs. The decoder structures with iterative decoding algorithms are presented in Section III. Physical layer of WiMAX is described in Section IV. Simulation results are in Section V. Finally, Section VI explains the conclusion and future work.

\section{ENCODER STRUCTURE IN SCCCS AND PCCCS}

Fig. 1 and Fig. 2 depict SCCCs, PCCCs encoder which are consisted of two convolutional encoders. They are separated by an interleaver. First and second encoders in SCCCs are called outer and inner encoder, respectively.

In SCCCs, information is entered into the outer encoder, encoded with $1 / 2$ code rate and next sent to the interleaver. Outputs of interleaver are sent to the inner encoder and encoded with $2 / 3$ code rate. Final code rate of information in SCCC is $1 / 3(1 / 2 \times 2 / 3=1 / 3)$. As well in PCCCs, information entered to the encoder for systematic production and two parity sequences.

Quality of coding in SCCCs and PCCCs depend on coding parameters and interleaver. Suitable parameters of convolutional codes for encoder and interleaver are presented in [5], [6].

\section{DECODER STRUCTURE IN SCCCS AND PCCCS WITH ITERATIVE DECODING ALGORITHM}

Iterative algorithm based on a posteriori probabilities later called as the BCJR, Maximum a posteriori (MAP) is used for decoding in SCCCs and PCCCs. The algorithm is simplified to the log-MAP and the Max-log-MAP algorithms [7]. Iterative decoding algorithm is carried out by exchanging of estimates of the information bits between two Soft-Input/Soft-Output (SISO) decoders as depicted in Fig. 3 and Fig. 4. This algorithm prepares an estimation of coded information bits measured based on their log-likelihood ratio by [8].

$$
L\left(u_{k}\right)=\ln \frac{P\left(u_{k}=+1\right)}{P\left(u_{k}=-1\right)}
$$

where $u_{k}$ is the input information bit at time $\mathrm{k}$. 


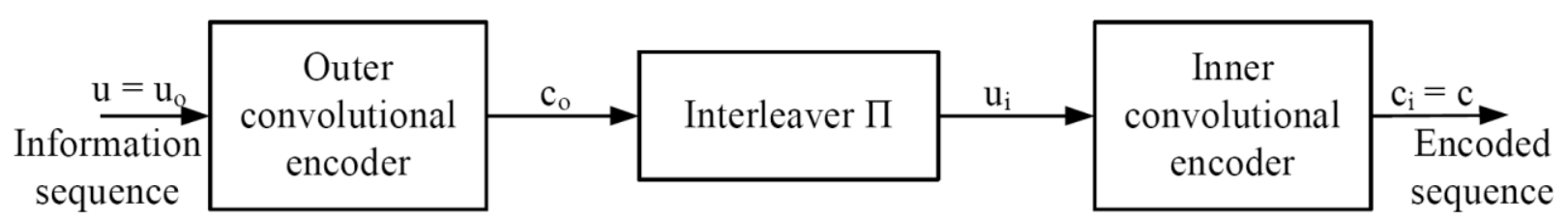

Fig. 1. Structure of encoder in serial concatenated convolutional codes [9].

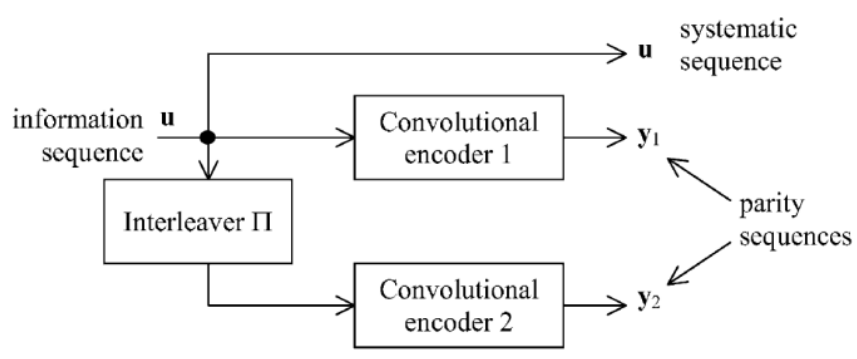

Fig. 2. Structure of encoder in parallel concatenated convolutional codes [9].

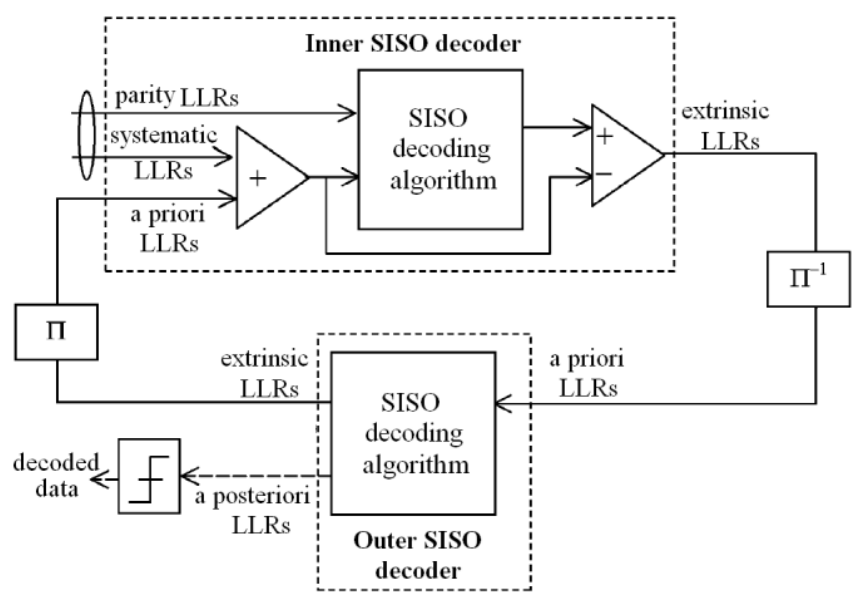

Fig. 3. Structure of decoder in serial concatenated convolutional codes [9].

\section{A. SCCCs Decoder}

Fig. 3 depicts SCCCs decoder which is consisted of two SISO or inner and outer decoders. The inner decoder receives the channel sequence containing the received information of the transmitted systematic bits, parity bits and a priori LLR. At the first iteration a priori LLR, $L\left(u_{k}\right)$ is set to zero. After a mathematical computation of algorithm [7], a posteriori LLR, $L\left(u_{k} \mid y\right)$ can be computed by

$$
L\left(u_{k} \mid y\right)=L\left(u_{k}\right)+L_{c} y_{k 1}+L_{e}\left(u_{k}\right)
$$

where first two terms in (2) depend on input information $u_{k}$, the third term i.e. the extrinsic LLRs, $L_{e}\left(u_{k}\right)$ are related to the codeword parity bits. $L_{e}\left(u_{k}\right)$, is computed by (3) and considered as a priori LLRs, $L\left(u_{k}\right)$ for outer decoder i.e. $L\left(u_{k}\right)=L_{e}\left(u_{k}\right)$.

$$
L_{e}\left(u_{k}\right)=L\left(u_{k} \mid y\right)-L\left(u_{k}\right)-L_{c} y_{k l}
$$

The outer decoder receives deinterleaved information of the transmitted systematic bits, parity bits and a priori LLRs. By using algorithm and (2) again, a posteriori LLRs are computed. Then information bits, $u_{k}$ can be estimated by determination sign of a posteriori LLR with hard decision.

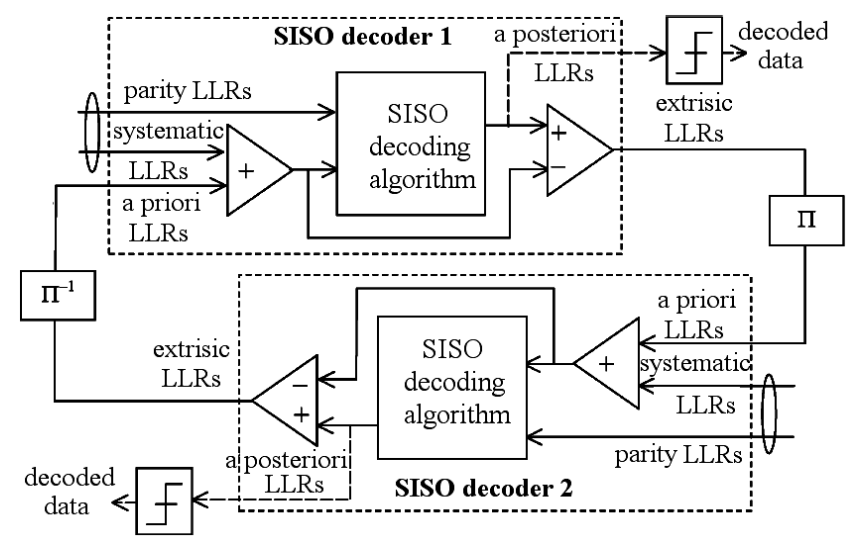

Fig. 4. Structure of decoder in parallel concatenated convolutional codes [9].

The decoder estimates bit $u_{k}=+1$ as input information when $L\left(u_{k} \mid y\right)>0$. Likewise, it estimates $u_{k}=-1$ if $L\left(u_{k} \mid y\right)<0$. Again $L_{e}\left(u_{k}\right)$ are computed by (3) and entered to the interleaver, then sent to the inner decoder, for second iteration. Here is the end of first iteration.

\section{B. PCCCs Decoder}

Fig. 4 depicts PCCCs decoder which is constructed of two SISO decoders. First decoder receives systematic bits and parity bits and a priori LLRs. As said, in the first iteration a priori LLR are set to zero. By applying mathematical computation of algorithm and (2), a posteriori LLRs, $L\left(u_{k} \mid y\right)$ are computed. As before said, by determining sign of a posteriori LLRs with hard decision, information of the first encoder are decoded. $L_{e}\left(u_{k}\right)$ is computed by (3) and sent to the interleaver and presumed as a priori LLR, $L\left(u_{k}\right)$ for second decoder.

Second decoder receives interleaved a priori LLRs, systematic bits and parity bits. By using (2) and algorithm, a posteriori LLRs are computed. Then information bits can be estimated by determining sign of a posteriori LLRs with hard decision. Again $L_{e}\left(u_{k}\right)$ are computed by (3) and entered to the deinterleaver and sent to the first decoder for second iteration. This is the end of iteration in PCCCs. This algorithm is repeated for several iterations, as mentioned for both of SCCCs and PCCCs. Consequently, with each iteration the bit error rate (BER) decreases and performance becomes better and better. In order to decrease decoding complexity, usually algorithm is carried out only about six or eight iterations. 


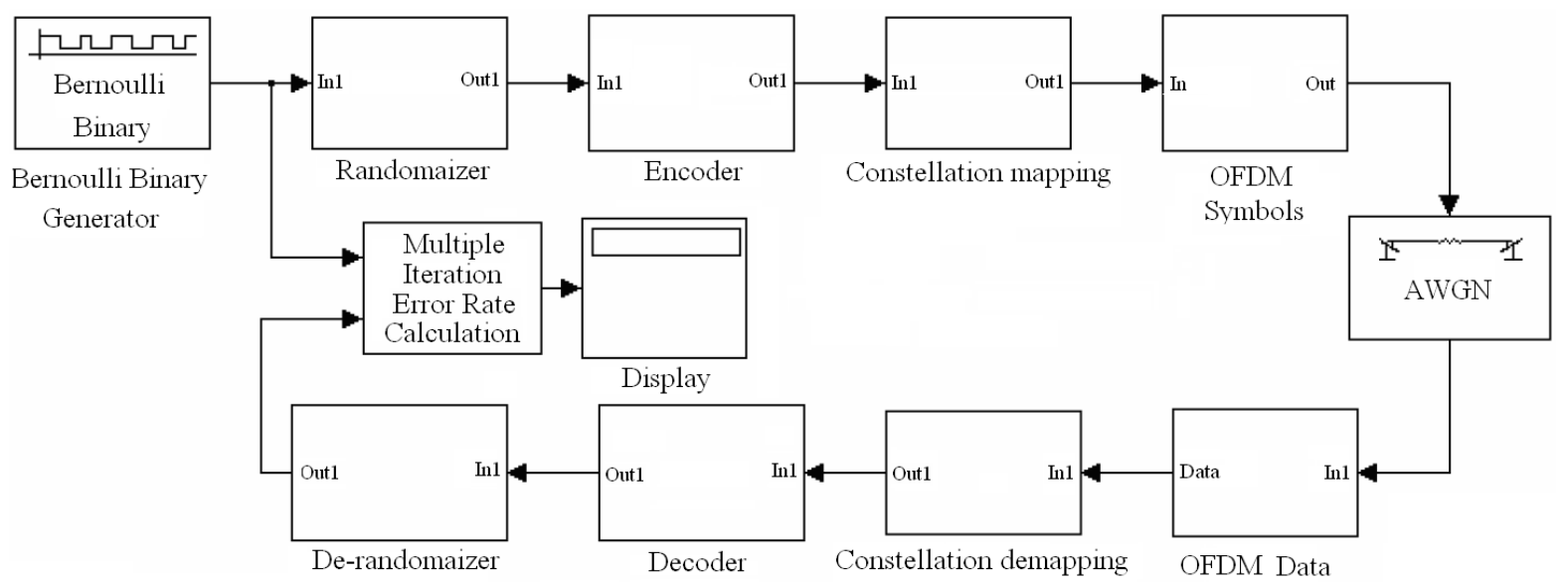

Fig. 5. Structure of physical layer of WiMAX.

\section{Physical Layer of WiMAX System}

In this paper physical layer of WiMAX simulated in MATLAB which is shown in Fig. 5.

\section{A. Transmitter}

Transmitter is composed as follows [2]:

- Bernoulli Binary Generator for generation input information.

- Randomizer for prevention of transmission iterative zero and one.

- Encoders which are used in this paper: SCCC, PCCC and RS-CC.

- Constellation mapping, it is caused coded information mapped to determined phase and amplitude. Standard offered BPSK, QPSK, 16QAM and 64QAM for modulation. It is useful to decrease error of transmitted bits and correct deviation from real amount of bits easily.

- Orthogonal Frequency Division Multiplexing (OFDM) is a multicarrier modulation, which uses Inverse Fast Fourier Transform (IFFT) to produce orthogonal sub-carriers for data modulation.

\section{B. Channel}

In this paper, Additive White Gaussian Noise (AWGN) channel is considered.

\section{Receiver}

Receiver is composed of components which invert actions of transmitter, i.e. OFDM demodulation which uses Fast Fourier Transform (FFT), constellation demapping, decoder and derandomizer [2].

\section{Simulation Results}

At first, physical layer of WiMAX is simulated by RS-CC, SCCC and PCCC in MATLAB software with parameters which are shown in TABLE I, II, and III, respectively. The Viterbi algorithm is considered for RS-CC decoding. The Max-Log-MAP algorithm is used for SCCC and PCCC decoding. This work is implemented with BPSK and 64QAM modulations. Then results are compared with each other and performance is defined based on Bit Error Rate (BER) in terms of $\mathrm{Eb} / \mathrm{N} 0$.

TABLE I: PARAMETERS OF RS-CC.

\begin{tabular}{|l|c|c|}
\hline Reed Solomon(RS) & \multicolumn{2}{|c|}{ Convolution code (CC) } \\
\hline $\mathrm{N}=255$ & Code rate & $\mathrm{R}=1 / 2$ \\
\hline $\mathrm{K}=239$ & $\begin{array}{c}\text { Generator } \\
\text { polynomials }\end{array}$ & $\mathrm{G} 1=171 \quad \mathrm{G} 2=133$ \\
$\mathrm{~T}=8$ & Constraint length & $\mathrm{L}=7$ \\
\hline
\end{tabular}

TABLE II: PARAMETERS OF SCCC.

\begin{tabular}{|c|c|c|c|c|}
\hline $\begin{array}{c}\text { Type } \\
\text { of } \\
\text { Encoder }\end{array}$ & $\begin{array}{c}\text { Constraint } \\
\text { length }\end{array}$ & $\begin{array}{c}\text { Generator } \\
\text { matrix }\end{array}$ & $\begin{array}{c}\text { Feedback } \\
\text { connection }\end{array}$ & $\begin{array}{c}\text { Code } \\
\text { rate }\end{array}$ \\
\hline Outer & 3 & {$[75]$} & 7 & $1 / 2$ \\
\hline Inner & {$[33]$} & {$[705 ; 076]$} & {$[77]$} & $2 / 3$ \\
\hline
\end{tabular}

TABLE III: PARAMETERS OF PCCC.

\begin{tabular}{|c|c|c|c|}
\hline $\begin{array}{c}\text { Constraint } \\
\text { length }\end{array}$ & $\begin{array}{c}\text { Generator } \\
\text { matrix }\end{array}$ & $\begin{array}{c}\text { Feedback } \\
\text { connection }\end{array}$ & Code rate \\
\hline 3 & {$[75]$} & 7 & $1 / 3$ \\
\hline
\end{tabular}

Fig. 6, Fig. 7, Fig. 8 and Fig. 9 show that BER decreases considerably when number of iterations increases from 1 to 6 in iterative decoding algorithm for SCCC and PCCC. Iterative decoding algorithm is an effective and strong way for decoding in SCCC and PCCC in physical layer of WiMAX. For lower Eb/NO, BPSK gives better performance than 64QAM in WiMAX. So, BPSK is used when channel have bad conditions and both of them (BPSK and 64QAM) can be used in high Eb/NO. Fig. 10 and Fig. 11 show using of SCCC and PCCC as FEC in physical layer of WiMAX, lead to better performance than RS-CC. 


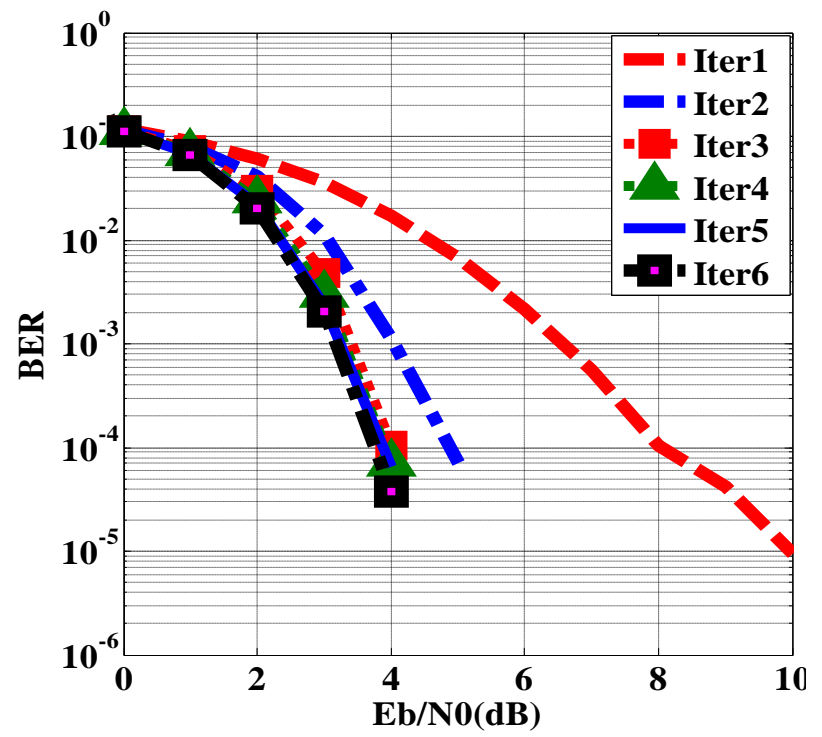

Fig. 6. BER versus Eb/N0 for BPSK in SCCC, Iteration=6.

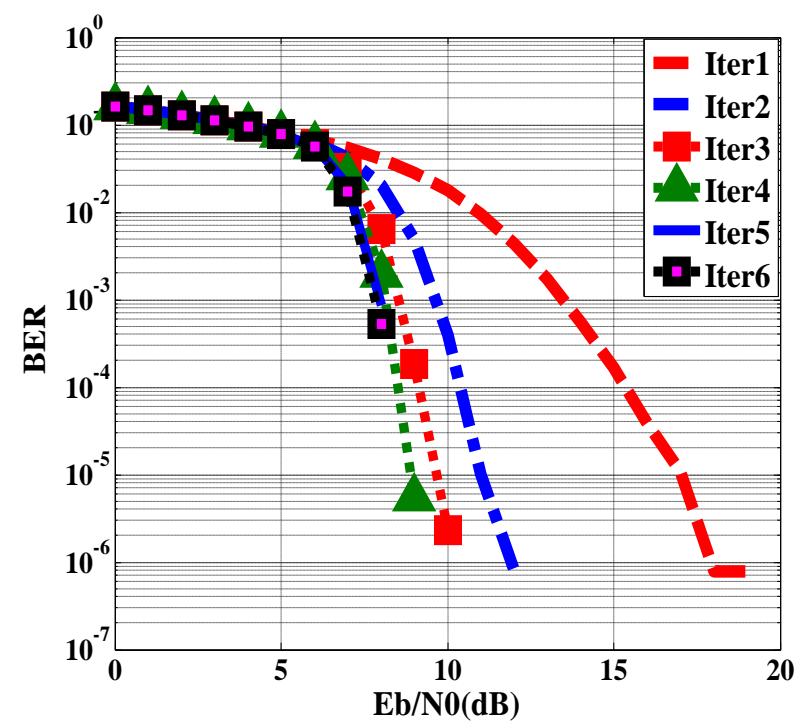

Fig. 7. BER versus Eb/N0 for 64QAM in SCCC, Iteration=6.

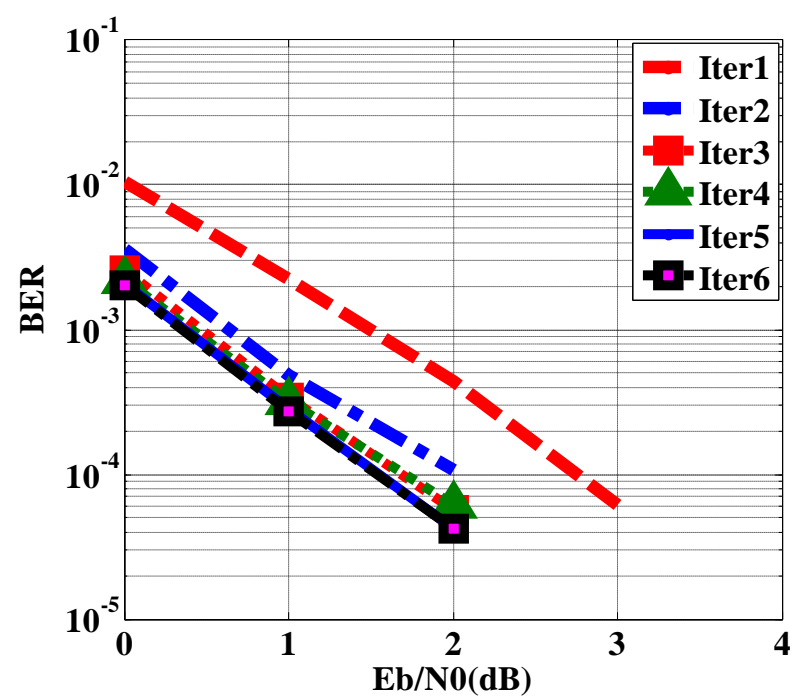

Fig. 8. BER versus Eb/N0 for BPSK in PCCC, Iteration=6.

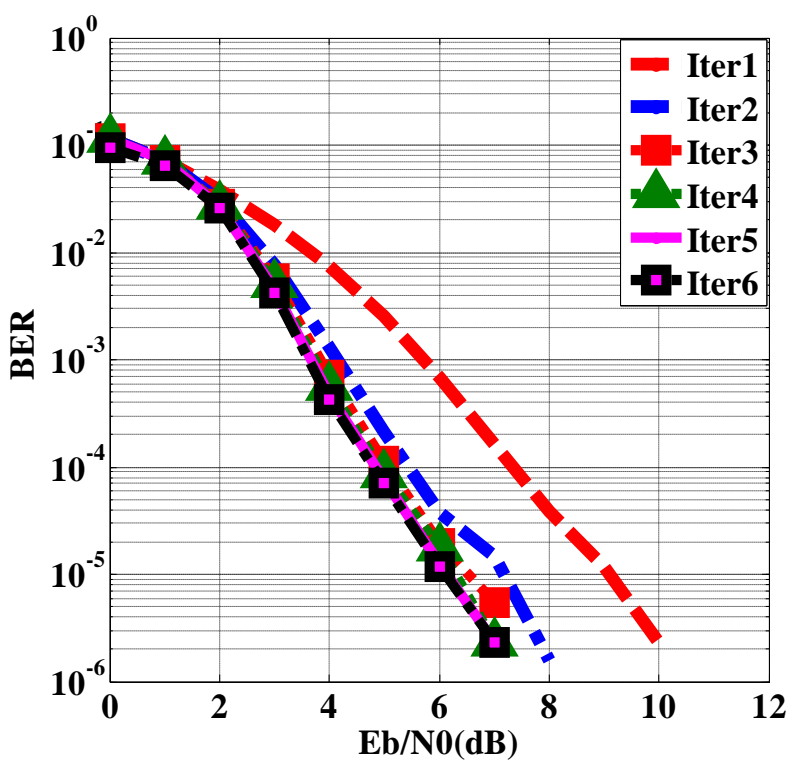

Fig. 9. BER versus Eb/N0 for 64QAM in PCCC, Iteration=6.

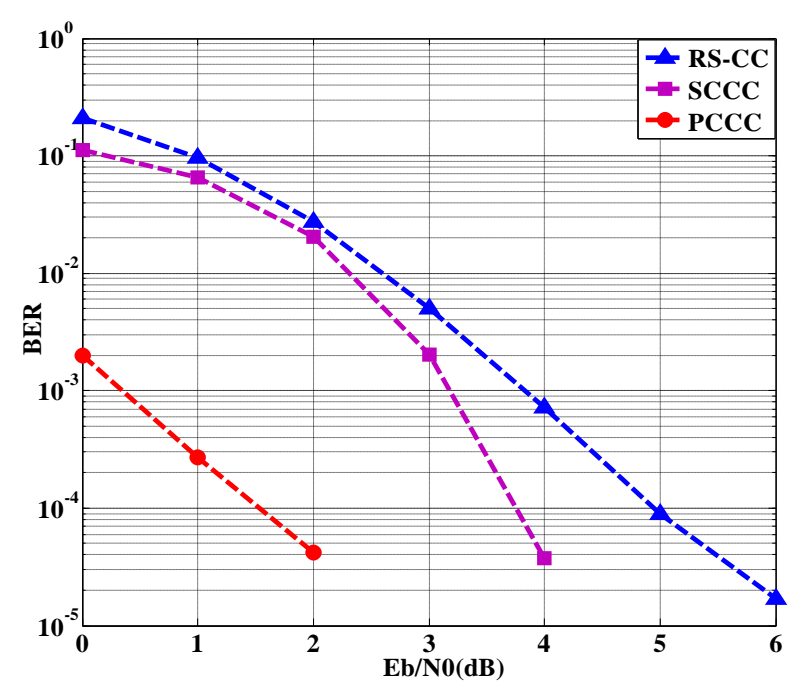

Fig. 10. BER versus Eb/N0 for BPSK in RS-CC, SCCC, PCCC.

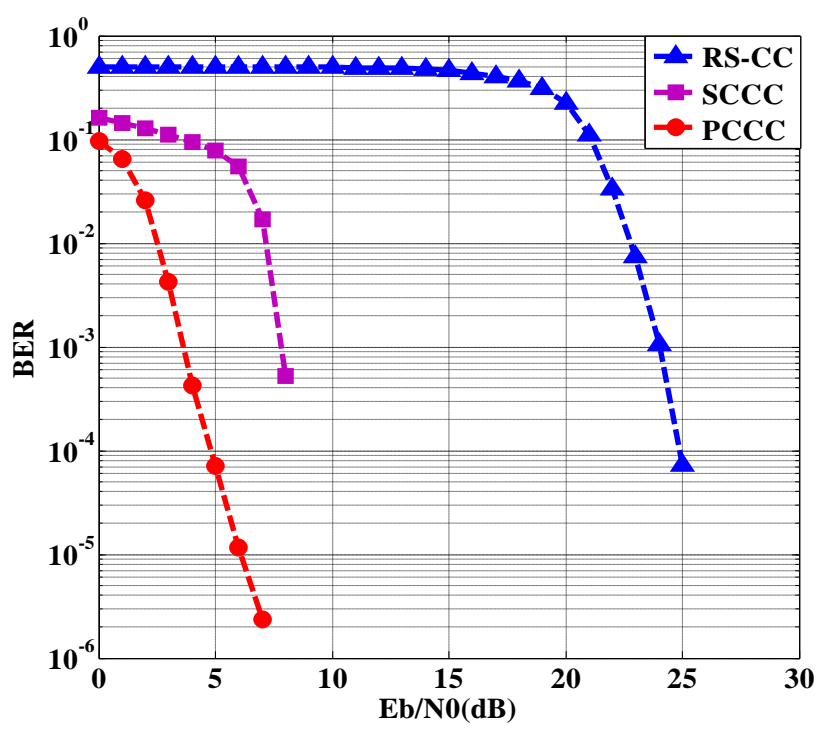

Fig. 11. BER versus Eb/N0 for 64QAM in RS-CC, SCCC, PCCC. 


\section{CONCLUSION}

WiMAX performance is improved by using SCCC, PCCC as FEC. Results show that SCCC and PCCC had better performance than RS-CC because of their superior coding and iterative decoding algorithms in this work. Using BPSK and 64QAM modulation with FEC were effective in various conditions of channel in terms of Eb/N0. Future work will involve investigating about fading channel, structure of interleaver and types of generator matrices to try in physical layer of WiMAX.

\section{REFERENCES}

[1] IEEE Computer Society, "Part 16: Air interface for fixed broadband wireless access systems," IEEE Standard for Local and Metropolitan Area Networks, IEEE 802.16-2004, New York, Nov. 2004.

[2] G. A. Jeffrey, G. Arunabha, and M. Rias, "Fundamentals of WiMAX, 2007 United States of America," Prentice Hall, pp. 62-92, 2007.

[3] P. Elias, "Error-free coding," IRE Transaction on Information Theory, vol. 4, no. 4, pp. 29-37, 1954

[4] D. Forney, "Concatenated Codes," Cambridge, MA: MIT Press, 1966.

[5] C. Berrou, A. Glavieux, and P. Thitimajshima, "Near Shannon limit error-correcting coding and decoding: Turbo-codes," in Proc. IEEE ICC, Geneva, Switzerland, pp. 1064-1070, May 1993.

[6] D. Divsalar and F. Pollara, "Multiple Turbo codes for deep-space communications," TDA Progress, Report, Jet Propulsion Laboratory, Report 42-121, pp. 66-77, May 1995.

[7] L. Hanzo, T. H. Liew, and B. L. Yeap, "Turbo coding, turbo equalisation and space-time coding for transmission over fading channels," New York: Wiley, 2002.

[8] J. Ortin, P. Garcia, F. Gutierrez, and A. Valdovinos, "Performance analysis of turbo decoding algorithms in wireless OFDM systems," IEEE Transactions on Consumer Electronics, vol. 55, no. 3, pp. 11491154, July 2009.
[9] E. Boutillon, C. Douillard, and G. Montorsi, "Iterative decoding of concatenated Convolutional codes: Implementation issues,' Proceedings of the IEEE, vol. 95, no. 6, pp. 1201-1227, June 2007.

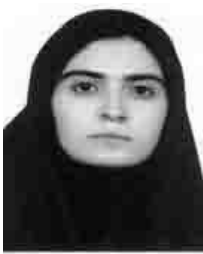

Parisa Rastegari received the B.S. and M.S. degrees in electrical engineering from the Islamic Azad University, Najafabad Branch, Isfahan, Iran, in 2006 and 2011, respectively. Her research interests include digital communications, wireless communications, and channel coding.

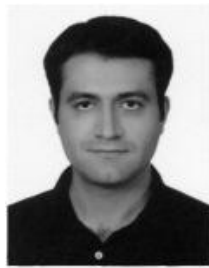

Mahdi Majidi received the B.S. degree in electrical engineering from Isfahan University of Technology, Isfahan, Iran, in 2004, and the M.S. degree in electrical engineering from Amirkabir University of Technology, Tehran, Iran, in 2007. He is currently pursuing the Ph.D. degree in electrical engineering at Amirkabir University of Technology. His research interests include nonlinear optimization methods, cognitive radio networks, and advanced signal processing.

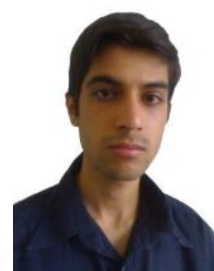

Mojtaba Khalilian was born in Isfahan, Iran, in 1985. He received the B.S. and M.S. degrees in electrica engineering from the Islamic Azad University, Najafabad Branch, Isfahan, Iran, in 2007 and 2011 respectively. His research interests include modeling and simulation of power converter and electrical machinery. 